PROCEEDINGS OF THE

AMERICAN MATHEMATICAL SOCIETY

Volume 136, Number 7, July 2008, Pages 2583-2587

S 0002-9939(08)09227-7

Article electronically published on February 29, 2008

\title{
SMOOTH APPROXIMATION OF DEFINABLE CONTINUOUS FUNCTIONS
}

\author{
ANDREAS FISCHER
}

(Communicated by Julia Knight)

\begin{abstract}
Let $\mathcal{M}$ be an o-minimal expansion of the real exponential field which possesses smooth cell decomposition. We prove that for every definable open set, the definable indefinitely continuously differentiable functions are a dense subset of the definable continuous function with respect to the o-minimal Whitney topology.
\end{abstract}

\section{INTRODUCTION}

In 3 and 6 it was shown that semialgebraic continuous functions can be approximated by Nash functions with respect to the semialgebraic Whitney topology. Similar approximations were studied for o-minimal structures.

We assume the reader to be familiar with the basic concepts of o-minimal structures, as they are presented in [1] or [2]. In the sequel, "definable" always means "definable with parameters in $\mathcal{M}$ " for a given o-minimal structure $\mathcal{M}$. Let $\mathbb{R}^{n}$ be endowed with the Euclidean topology. For a definable open set $U$ and $p \in \mathbb{N} \cup\{\infty\}$, we denote by $\mathcal{C}^{p}\left(U, \mathbb{R}^{k}\right)$ the definable $p$ times continuously differentiable functions from $U$ to $\mathbb{R}^{k}$, and by $\mathcal{C}(U, \mathbb{R})$ the definable continuous functions from $U$ to $\mathbb{R}$.

The o-minimal Whitney topology on $\mathcal{C}(U, \mathbb{R})$ is the coarsest topology for which the sets

(1.1) $\{g \in \mathcal{C}(U, \mathbb{R}):|f(u)-g(u)|<\varepsilon(u), u \in U\}, f \in \mathcal{C}(U, \mathbb{R}), \varepsilon \in \mathcal{C}(U,(0, \infty))$,

form a basis. In all $o$-minimal expansions of the reals, $\mathcal{C}^{p}(U, \mathbb{R}) \subset \mathcal{C}(U, \mathbb{R})$ is dense with respect to the $o$-minimal Whitney topology if $p<\infty$; cf. 4 .

Here we consider $o$-minimal expansions $\mathcal{M}$ of the real exponential field. In 5 , Chap. 8] it was shown that for every definable open set $U \subset \mathbb{R}^{n}, \mathcal{C}^{\infty}(U, \mathbb{R}) \subset$ $\mathcal{C}(U, \mathbb{R})$ is dense with respect to the $o$-minimal Whitney topology if (a) $\mathcal{M}$ is locally polynomially bounded, and (b) $\mathcal{M}$ possesses $\mathcal{C}^{\infty}$ cell decomposition.

By proving the following theorem, we show that assumption (a) can be omitted.

Theorem 1.1. Let $\mathcal{M}$ be an o-minimal expansion of the real exponential field which possesses $\mathcal{C}^{\infty}$ cell decomposition. Let $U \subset \mathbb{R}^{n}$ be open and let $f \in \mathcal{C}(U, \mathbb{R})$. Then, for every $\varepsilon \in \mathcal{C}(U,(0, \infty))$, there is a $g \in \mathcal{C}^{\infty}(U, \mathbb{R})$ with $|g(u)-f(u)|<\varepsilon(u), u \in U$.

Received by the editors January 31, 2007, and, in revised form, April 10, 2007, and May 15, 2007.

2000 Mathematics Subject Classification. Primary 03C64; Secondary 26E10.

Key words and phrases. o-minimal structures, exponential function, approximation.

This research was partially supported by the NSERC discovery grant of Dr. Salma Kuhlmann.

(C)2008 American Mathematical Society

Reverts to public domain 28 years from publication 


\section{Preliminaries}

A definable function $f: A \rightarrow \mathbb{R}^{k}$ is called a $\mathcal{C}^{\infty}$ function, if there are an open definable neighborhood $U$ of $A$ and an $F \in \mathcal{C}^{\infty}\left(U, \mathbb{R}^{k}\right)$ such that $\left.F\right|_{A}=f$.

Definition 2.1. A $\mathcal{C}^{\infty}$ cell in $\mathbb{R}$ is either a single point or an open interval. Supposing all cells of $\mathbb{R}^{n}$ are known, then a $\mathcal{C}^{\infty}$ cell in $\mathbb{R}^{n+1}$ is a set of either the form $(h)_{X}:=\{(x, r): x \in X, r=h(x)\}$ where $X \subset \mathbb{R}^{n}$ is a $\mathcal{C}^{\infty}$ cell and $h \in \mathcal{C}^{\infty}(X, \mathbb{R})$, or of the form $(f, g)_{X}:=\{(x, r): x \in X, f(x)<r<g(x)\}$ where $X \subset \mathbb{R}^{n}$ is a $\mathcal{C}^{\infty}$ cell and $f, g \in \mathcal{C}^{\infty}(X, \mathbb{R}) \cup\{ \pm \infty\}$ such that $f(x)<g(x), x \in X$.

The functions used to describe a $\mathcal{C}^{\infty}$ cell $Z$ we call the defining functions of $Z$.

Note that a $\mathcal{C}^{\infty}$ cell decomposition of $\mathbb{R}$ is a finite partition of $\mathbb{R}$ into $\mathcal{C}^{\infty}$ cells. A finite partition of $\mathbb{R}^{n+1}$ into $\mathcal{C}^{\infty}$ cells $Z_{1}, \ldots, Z_{r}$ is called a $\mathcal{C}^{\infty}$ cell decomposition, if the set of projections $\pi\left(Z_{i}\right), i=1, \ldots, r$, is a $\mathcal{C}^{\infty}$ cell decomposition of $\mathbb{R}^{n}$, where $\pi$ is the projection onto the first $n$ coordinates.

From now on we assume that $\mathcal{M}$ possesses $\mathcal{C}^{\infty}$ cell decomposition. That is, for any finite collection of definable sets $A_{1}, \ldots, A_{k} \subset \mathbb{R}^{n}$ there exists a $\mathcal{C}^{\infty}$ cell decomposition of $\mathbb{R}^{n}$ partitioning each $A_{i}, i=1, \ldots, k$.

So far, all known $o$-minimal structures have this property. For a set $Z, \operatorname{cl}(Z)$ denotes its topological closure, and $\partial Z:=\operatorname{cl}(Z) \backslash Z$ its frontier.

Lemma 2.2. Let $Z \subset \mathbb{R}^{n}$ be a bounded open $\mathcal{C}^{\infty}$ cell and let $f \in \mathcal{C}(\operatorname{cl}(Z),[0, \infty))$ satisfy $f>0$ on $Z$. Then there is a $g \in \mathcal{C}^{\infty}(Z, \mathbb{R})$ such that

$$
0<g(z)<f(z), z \in Z \text {. }
$$

Proof. Let $f_{i}, g_{i}, i=1, \ldots, n$, be the defining functions of $Z$. Then $\rho: Z \rightarrow \mathbb{R}$,

$$
\rho\left(x_{1}, \ldots, x_{n}\right):=\prod_{i=1}^{n}\left(x_{i}-f_{i}\left(x_{1}, \ldots, x_{i-1}\right)\right)\left(g_{i}\left(x_{1}, \ldots, x_{i-1}\right)-x_{i}\right),\left(x_{1}, \ldots, x_{n}\right) \in Z,
$$

is a $\mathcal{C}^{\infty}$ function, which is positive on $Z$ and extends to a definable continuous function $\bar{\rho}: \operatorname{cl}(Z) \rightarrow \mathbb{R}$ with $\bar{\rho}(z)=0, z \in \partial Z$.

We apply a generalized Łojasiewicz inequality (cf. 2, C.14]) to $\bar{\rho}, f$ and $\operatorname{cl}(Z)$, and obtain a definable continuous strictly monotone function $\phi: \mathbb{R} \rightarrow \mathbb{R}$ with $\phi(0)=0$ such that $0<\phi(\bar{\rho}(z)) \leq f(z), z \in Z$.

By $\mathcal{C}^{\infty}$ cell decomposition, $\phi$ restricted to $(0, \delta)$ is a $\mathcal{C}^{\infty}$ function for some $\delta \in$ $(0,1)$. Let $\psi: \mathbb{R} \rightarrow \mathbb{R}$ be defined by $\psi(t):=\phi\left(\delta t^{2} /\left(1+t^{2}\right)\right)$. Then $g:=\psi \circ \rho: Z \rightarrow \mathbb{R}$ is a $\mathcal{C}^{\infty}$ function and satisfies inequality (2.1), as $\delta<1$.

\section{Proof of Theorem 1.1}

Proof of Theorem 1.1. By applying the function $\tau: \mathbb{R}^{n} \rightarrow \mathbb{R}^{n}$ which is defined by $\tau\left(x_{1}, \ldots, x_{n}\right)=\left(x_{1} / \sqrt{1+x_{1}^{2}}, \ldots, x_{n} / \sqrt{1+x_{n}^{2}}\right)$ we can reduce our consideration to bounded open sets.

By $\mathcal{C}^{\infty}$ cell decomposition there are finitely many disjoint $\mathcal{C}^{\infty}$ cells $Z_{1}, \ldots, Z_{s}$, which cover $U$ such that $\left.f\right|_{Z_{i}}$ is a $\mathcal{C}^{\infty}$ function, $i=1, \ldots, s$. This partition can be refined in such a way that $\partial Z_{i}$ is the union of some of the $\mathcal{C}^{\infty}$ cells; cf. [1, Chap. 4, Prop. 1.13]. Therefore, each $\mathcal{C}^{\infty}$ cell $Z_{i}$ has a definable open neighborhood $U_{i}$, which is disjoint to all cells $Z_{j}$, for $j \neq i$ and $\operatorname{dim}\left(Z_{j}\right) \leq \operatorname{dim}\left(Z_{i}\right)$. Let $Z_{1}, \ldots, Z_{q}$ be the cells of dimension less than $n$. We order these cells, such that $\operatorname{dim}\left(Z_{i+1}\right) \geq$ $\operatorname{dim}\left(Z_{i}\right), i=1, \ldots, q-1$. 
We prove the following statement, which implies the conclusion of Theorem 1.1 . by induction on $r$.

For all $\tilde{\varepsilon} \in \mathcal{C}(U,(0, \infty))$ and $F \in \mathcal{C}(U, \mathbb{R})$, which satisfy the conditions

(a) $\left.F\right|_{Z_{i}}$ is $\mathcal{C}^{\infty}$ smooth, $i=1, \ldots, r$, and

(b) $F$ is $\mathcal{C}^{\infty}$ smooth in $U \backslash \bigcup_{i=1}^{r} Z_{i}$,

there is a $g \in \mathcal{C}^{\infty}(U, \mathbb{R})$ such that $|g(u)-F(u)|<\tilde{\varepsilon}(u), u \in U$.

The case $r=0$ is evident. We assume that the statement holds for $r \geq 0$.

Let $Z:=Z_{r+1}$. After some permutation of the coordinates, $Z$ is the graph of a $\mathcal{C}^{\infty}$ function $h=\left(h_{d+1}, \ldots, h_{n}\right): X \rightarrow \mathbb{R}^{n-d}$ where $X \subset \mathbb{R}^{d}$ is some open $\mathcal{C}^{\infty}$ cell. There also exists an open definable neighborhood $U^{\prime}$ of $Z$ and a function $e \in \mathcal{C}^{\infty}\left(U^{\prime}, \mathbb{R}\right)$ with $\left.e\right|_{Z}=\left.F\right|_{Z}$. In addition, $U^{\prime}$ may be chosen so small that $U^{\prime} \cap Z_{i}=\emptyset, i=1, \ldots, r$, and $U^{\prime} \subset X \times \mathbb{R}^{n-d}$.

We take a definable open neighborhood $V$ of $Z$, which is contained in $U^{\prime}$ such that $|e(u)-F(u)|<\tilde{\varepsilon}(u) / 2, u \in V$. Let $\Delta: X \rightarrow \mathbb{R}$ be the definable continuous function, which maps $x \in X$ to

$$
\Delta(x):=\min \left(\operatorname{dist}\left((x, h(x)), \bigcup_{i \leq r} Z_{i}\right), \operatorname{dist}((x, h(x)), \partial Z), \operatorname{dist}((x, h(x)), \partial V)\right) .
$$

By Lemma 2.2 there is a $\varphi \in \mathcal{C}^{\infty}(X, \mathbb{R})$ such that $0<\varphi(x)<\Delta(x) / n, x \in X$. For $0<s \leq 1$, let $W_{s}$ be given by

$$
W_{s}:=\left\{(x, y): x \in X,\left|y_{i}-h_{i}(x)\right|<s \varphi(x), i=d+1, \ldots, n\right\} \subset V .
$$

The $W_{s}$ contain $Z$, and they are $\mathcal{C}^{\infty}$ cells with defining $\mathcal{C}^{\infty}$ functions $f_{i}, g_{i}$ of $X$, $i=1, \ldots, d$, and $f_{i}=h_{i}-s \varphi, g_{i}=h_{i}+s \varphi, i=d+1, \ldots, n$.

We let $\sigma: \mathbb{R} \rightarrow \mathbb{R}$ be the $\mathcal{C}^{\infty}$ function given by $\sigma(t)=\exp (-1 / t)$ if $t>0$, and $\sigma(t)=0$ if $t \leq 0$. Let the functions $\psi_{1}, \psi_{2}: X \times \mathbb{R}^{n-d} \rightarrow \mathbb{R}$ be defined by

$$
\begin{aligned}
& \psi_{1}(x, y):=\prod_{i=d+1}^{n} \sigma\left(y_{i}-h_{i}(x)+\varphi(x)\right) \sigma\left(h_{i}(x)+\varphi(x)-y_{i}\right), \text { respectively, } \\
& \psi_{2}(x, y):=\sum_{i=d+1}^{n}\left(\sigma\left(h_{i}(x)-\frac{1}{2} \varphi(x)-y_{i}\right)+\sigma\left(y_{i}-h_{i}(x)-\frac{1}{2} \varphi(x)\right)\right) .
\end{aligned}
$$

The functions $\psi_{1}$ and $\psi_{2}$ are definable $\mathcal{C}^{\infty}$ functions, and their sum is positive in $X \times \mathbb{R}^{n-d}$. Finally, we define the function $G: U \rightarrow \mathbb{R}$ by

$$
G(u):= \begin{cases}\frac{\psi_{1}(u) e(u)+\psi_{2}(u) F(u)}{\psi_{1}(u)+\psi_{2}(u)}, & \text { if } u \in V \\ F(u), & \text { otherwise. }\end{cases}
$$

The function $\psi_{1}$ vanishes outside of $W_{1}$, and $\psi_{2}$ equals zero in $W_{1 / 2}$. Hence, $G=F$ outside $W_{1}$, and $G=e$ in $W_{1 / 2}$. Moreover, $G \in \mathcal{C}(U, \mathbb{R})$, and $G$ satisfies

$$
|G(u)-F(u)|<\frac{1}{2} \tilde{\varepsilon}(u), u \in U .
$$

In addition, the set of points at which $G$ is not $\mathcal{C}^{\infty}$ smooth is contained in $\bigcup_{i=1}^{r} Z_{i}$, and $\left.G\right|_{Z_{i}}$ is a $\mathcal{C}^{\infty}$ function, $i=1, \ldots, r$, and $G$ restricted to $U \backslash \bigcup_{i=1}^{r} Z_{i}$ is $\mathcal{C}^{\infty}$ smooth. We apply the induction hypothesis to $\tilde{\varepsilon} / 2$ and $G$ in place of $\tilde{\varepsilon}$ and $F$ so that we obtain a $g \in \mathcal{C}^{\infty}(U, \mathbb{R})$ which satisfies $|g(u)-G(u)|<\tilde{\varepsilon}(u) / 2, u \in U$. Therefore,

$$
|g(u)-F(u)|<|g(u)-G(u)|+|G(u)-F(u)|<\tilde{\varepsilon}(u), u \in U .
$$


Remark 3.1. In the situation described in Theorem 1.1 let $S \subset U$ be a definable set that is closed in $U$ and contains the set of non-smooth points of $f$. If $W$ is any definable open neighborhood of $S$, we may assume that $g=f$ outside of $W$. This is due to the fact that in the proof of Theorem 1.1 we can select a $\mathcal{C}^{\infty}$ cell decomposition partitioning $S$, and for the induction we only have to consider the cells contained in $S$. The corresponding neighborhoods of the cells may be chosen as subsets of $W$.

\section{Consequences}

Theorem 1.1 implies a more general statement.

Corollary 4.1. Let $A \subset \mathbb{R}^{n}$ be a locally closed definable set, and let $f \in \mathcal{C}(A, \mathbb{R})$. Then for every $\varepsilon \in \mathcal{C}(A,(0, \infty))$ there is a definable open neighborhood $V$ of $A$ and a $g \in \mathcal{C}^{\infty}(V, \mathbb{R})$ such that $|g(a)-f(a)|<\varepsilon(a), a \in A$.

Proof. Since $A$ is locally closed, there are definable sets $B$ and $V$, where the former is closed and the latter is open, such that $A=B \cap V$. Take an $F \in \mathcal{C}(V, \mathbb{R})$ with $\left.F\right|_{A}=f$ and $\tilde{\varepsilon} \in \mathcal{C}(V,(0, \infty))$ with $\left.\tilde{\varepsilon}\right|_{A}=\varepsilon$; cf. [1, p. 138, Cor. 3.10]. By Theorem 1.1 there is a $g \in \mathcal{C}^{\infty}(V, \mathbb{R})$ such that $|g(v)-F(v)|<\tilde{\varepsilon}(v), v \in V$. Hence, $g$ has the desired properties.

The next consequence is $\mathcal{C}^{\infty}$ separation of sets.

Corollary 4.2. Let $A_{0}, \ldots, A_{k} \subset \mathbb{R}^{n}$ be disjoint closed definable sets. Then there is $a \phi \in \mathcal{C}^{\infty}\left(\mathbb{R}^{n}, \mathbb{R}\right)$ such that $A_{i} \subset \phi^{-1}(\{i\}), i=0, \ldots, k$.

Proof. Let $U_{i}$ be a definable open neighborhood of $A_{i}, i=0, \ldots, k$, such that $\operatorname{cl}\left(U_{i}\right) \cap$ $\operatorname{cl}\left(U_{j}\right)=\emptyset, i \neq j$. Select for each $i=1, \ldots, k$ a definable continuous function $g_{i}: \mathbb{R}^{n} \rightarrow[0,1]$ that vanishes in $\operatorname{cl}\left(U_{j}\right), j \neq i$, and that equals 1 in $\operatorname{cl}\left(U_{i}\right)$; cf. [1, p. 102, Lem. 3.8]. Then $g: \mathbb{R}^{n} \rightarrow \mathbb{R}$,

$$
g(u)=\sum_{i=1}^{k} i g_{i}(u), u \in U
$$

is definable and continuous such that $\operatorname{cl}\left(U_{i}\right) \subset\{x: g(x)=i\}, i=0, \ldots, k$. Then $\mathbb{R}^{n} \backslash\left(\bigcup_{i=0}^{k} A_{i}\right)$ is a definable open neighborhood of the points at which $g$ is not $\mathcal{C}^{\infty}$ smooth. By Theorem 1.1] in connection with Remark 3.1 there is an $f \in \mathcal{C}^{\infty}\left(\mathbb{R}^{n}, \mathbb{R}\right)$ with $f=g$ on $\bigcup_{i=0}^{k} A_{i}$.

Finally, we note the following corollary.

Corollary 4.3. Let $A \subset \mathbb{R}^{n}$ be a closed definable set, let $U \supset A$ be definable and open, and let $f \in \mathcal{C}^{\infty}(U, \mathbb{R})$. Then there is an $F \in \mathcal{C}^{\infty}\left(\mathbb{R}^{n}, \mathbb{R}\right)$ such that $\left.F\right|_{A}=\left.f\right|_{A}$.

Proof. Let $V$ be an open definable neighborhood of $A$ such that $\operatorname{cl}(V) \subset U$. Set $A_{0}:=\mathbb{R}^{n} \backslash V$ and $A_{1}:=A$. By Corollary 4.2 there is a $g \in \mathcal{C}^{\infty}\left(\mathbb{R}^{n}, \mathbb{R}\right)$ such that $A_{i} \subset\{x: g(x)=i\}$. Set $F(x):=f(x) g(x)$ if $x \in V$, and $F(x):=0$ otherwise.

\section{REFERENCES}

1. van den Dries, L., Tame topology and o-minimal structures. London Mathematical Society Lecture Note Series, 248. Cambridge University Press, Cambridge, 1998. MR1633348 (99j:03001)

2. van den Dries, L., and Miller, C., Geometric categories and o-minimal structures. Duke Math. J. 84 (1996), no. 2, 497-540. MR1404337 (97i:32008) 
3. Efroymson, G. A., The extension theorem for Nash functions. Real algebraic geometry and quadratic forms (Rennes, 1981), pp. 343-357, Lecture Notes in Math., 959, Springer, BerlinNew York, 1982. MR683141 (84i:58002)

4. Escribano, J., Approximation theorems in o-minimal structures, Illinois Journal of Mathematics 46(1) (2002), 111-128. MR.1936078 (2003i:03042)

5. Jones, G. O., Local to global methods in o-minimal expansions of fields. Doctoral Thesis, Wolfson College University of Oxford, 2006.

6. Pecker, D., On Efroymson's extension theorem for Nash functions. J. Pure Appl. Algebra 37 (1985), no. 2, 193-203. MR796409 (87e:58002)

Department of Mathematics and Statistics, University of Saskatchewan, 106 Wiggins Road, Saskatoon, SK, S7N 5E6, Canada

E-mail address: el.fischerandreas@web.de 